\title{
Lesional Temporal Lobe Epilepsy: Does the "Uncommon" Differ from the Common?
}

\author{
Ashutosh Kumar ${ }^{1}$ Suyash Singh ${ }^{2}$ Anant Mehrotra ${ }^{1} \quad$ Lily Pal $^{3}$ Shweta Katiyar ${ }^{3}$ \\ Nandita Chaudhary ${ }^{3}$ Pawan Kumar Verma ${ }^{1}$ Ved Prakash Maurya ${ }^{1}$ Kuntal K. Das ${ }^{1}$ \\ Jayesh Sardhara ${ }^{1}$ Kamlesh Singh Bhaisora ${ }^{1}$ Arun Kumar Srivastava ${ }^{1}$ Awadhesh Kumar Jaiswal ${ }^{1}$ \\ Sanjay Behari ${ }^{1}$ \\ ${ }^{1}$ Department of Neurosurgery, Sanjay Gandhi Postgraduate \\ Institute of Medical Sciences, Lucknow, Uttar Pradesh, India \\ ${ }^{2}$ Department of Neurosurgery, All India Institute of Medical \\ Sciences, Raebareli, Uttar Pradesh, India \\ ${ }^{3}$ Department of Pathology, Sanjay Gandhi Postgraduate Institute of \\ Medical Sciences, Lucknow, Uttar Pradesh, India \\ Indian | Neurosurg 2022;11:159-167

\begin{abstract}
Address for correspondence Anant Mehrotra, MCh, MS, MBBS, Department of Neurosurgery, Sanjay Gandhi Postgraduate Institute of Medical Sciences, Lucknow 226014, Uttar Pradesh, India (e-mail: dranantmehrotra@gmail.com).
\end{abstract}

\begin{abstract}
Keywords

- seizure outcome

- temporal lobe epilepsy

- pathology

- diffuse leptomeningeal glioneuronal tumor

- angiocentric glioma

- corticle ependymoma

Objectives This study aims to evaluate the subgroup consisting of uncommon pathological entities presenting with temporal lobe epilepsy (TLE).

Methodology Twenty-four consecutively operated patients of lesional temporal lobe epilepsy were included in the study. Eleven cases were identified that had pathologies implicated as "uncommon" in previously done studies on the pathology of TLE. A subgroup analysis consisting of these uncommon lesions was done for clinical presentation and seizure outcome. The seizure outcome was assessed in terms of Engel and International League Against Epilepsy (ILAE) classification.

Results Nine different pathological entities were identified within the study group. The mean age at surgery was 18.6 years (range $2-30$ years). The mean duration of epilepsy was 48.3 months and the average duration of follow-up was 39.2 months. All patients had seizures as the only complaint except three. Three patients had focal-aware seizures, two had focal motor onset, and the rest all had focal-unaware seizures. Seven patients were seizure free and the average age at diagnosis for these patients was 15.4 years (range 2-24 years.). The duration of seizure in the postoperative seizure-free group was 29.7 months and it was 81 months for the other group. All the patients with persistent postoperative seizures had focal-unaware seizures preoperatively.

Conclusion Despite the small and heterogeneous nature of this subgroup of uncommon lesions causing temporal lobe epilepsy, the clinical presentation, prognostic factors, and seizure outcome are similar to the cohort of common pathologies, including mesial temporal sclerosis.
\end{abstract}

published online February 4, 2022
DOI https://doi.org/ 10.1055/s-0041-1730128 ISSN 2277-954X
(C) 2022. Neurological Surgeons' Society of India.

This is an open access article published by Thieme under the terms of the Creative Commons Attribution-NonDerivative-NonCommercial-License, permitting copying and reproduction so long as the original work is given appropriate credit. Contents may not be used for commercial purposes, or adapted, remixed, transformed or built upon. (https://creativecommons.org/licenses/by-nc-nd/4.0/). Thieme Medical and Scientific Publishers Pvt. Ltd. A-12, 2nd Floor, Sector 2, Noida-201301 UP, India 


\section{Introduction}

Temporal lobe lesions are the most common cause for focal-onset seizures. ${ }^{1}$ They are implicated in more than $60 \%$ of adult-onset seizures. Mesial temporal sclerosis (MTS) is the most common cause of temporal lobe epilepsy. ${ }^{2,3}$ Apart from this, there is a heterogeneous group of structural lesions that cause temporal lobe epilepsy. It includes both neoplastic and non-neoplastic lesions. Within the neoplastic group, ganglioglioma and glioma are the most common pathological cause. The lesser of this group has been rarely explored as a subgroup. This uncommon subgroup includes some rare and few newly recognized pathologies. The focus of this article is on these uncommon causes of temporal lobe epilepsy. Despite the pathological heterogeneity, this subgroup has a striking similarity to the overall neoplastic group in terms of clinical presentation and seizure outcome.

\section{Methodology}

A total of 24 consecutively operated patients $(n=24)$ of lesional temporal lobe epilepsy were included in our study. These patients had undergone lesionectomy alone (LA) or lesionectomy with anteromedial temporal lobe resection (L + AMTR) for structural lesions of the temporal lobe. The patients were included irrespective of age and sex, who presented in our department with seizures as their primary complaint in the last 5 years (2015-2020). Cases having major tumor mass in temporal lobe with some extension to nearby structures were also included. These patients were on appropriate antiepileptic drugs (AED) given in adequate dose and duration. They were classified as medically intractable seizures or drug-resistant epilepsy based on International League Against Epilepsy (ILAE) guidelines. ${ }^{4}$ Magnetic resonance imaging (MRI) brain (in seizure protocol) was done as the initial investigation. Contrast-enhanced (gadolinium) MRI was done in cases with suspected tumors. In cases having more than one lesion, if seizure semiology was unclear from the history, or if the semiology was discordant with MRI findings, video electroencephalography
(EEG) or other noninvasive or invasive investigations were planned for correct lateralization and localization of seizure foci. Lesionectomy alone (LA) or lesionectomy with anteromedial temporal resection ( $\mathrm{L}+$ AMTR) was done based on the findings of the epilepsy workup. Electrocorticography (ECoG) was employed intraoperatively in deeply situated lesions. The histopathology was reviewed by an experienced neuropathologist. The pathologies that have been reported as uncommon causes of temporal lobe epilepsy were selected for subgroup analysis $(n=11) .^{5-7}$ Antiepileptics were not tapered in the immediate postoperative period. Once discharged, patients were followed-up in the outpatient department (OPD) and then the AED and their doses were modified to taper gradually and then stopped. The number of antiepileptic drugs and their doses, clinical profile, the surgical intervention done, and immediate postoperative course were recorded from the patient case files and the hospital information system (HIS) retrospectively. The data of clinical course following discharge was acquired from the follow-up records and through telephonic conversation with the patients. The seizure outcome was reported in terms of "Engel" and "ILAE" classification.

\section{Results}

Among the 24 consecutively operated patients, there were 5 cases of MTS; 11 cases of low-grade astrocytoma; 2 of leptomeningeal glioneuronal tumors; and 1 case each of cavernoma, epidermoid cyst, arachnoid cyst, angiocentric glioma ( - Fig. 1A, B), cortical ependymoma, and dysplastic neuroectodermal tumor (DNET). The 11 cases of low-grade glioma included diffuse astrocytoma (5), pleomorphic xanthoastrocytoma (2), anaplastic astrocytoma (1), pilocytic astrocytoma (1), pilomyxoid astrocytoma (1), and oligodendroglioma (1). Out of these, a subgroup of 11 cases was identified as having pathologies that have been less frequently described to present with temporal lobe epilepsy ${ }^{5-7}$ (- Table 1). We analyzed this subgroup for clinical presentation and seizure outcome. The mean age at surgery was 18.6 years with an age range of 2 to 30 years. The mean duration of epilepsy was 48.3 months

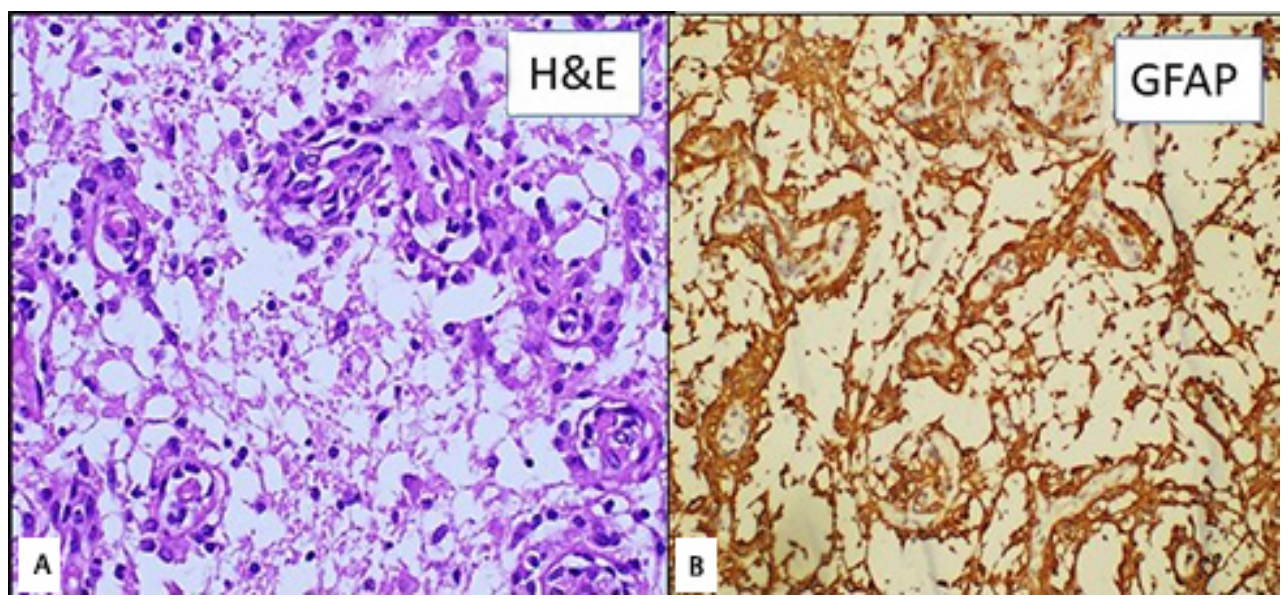

Fig. 1 (A) Angiocentric glioma with the perivascular arrangement of monomorphic glial cells. (B) Glial fibrillary acidic protein (GFAP) positivity in angiocentric glioma (magnification $\times 20$ ). 
Lesional Temporal Lobe Epilepsy Kumar et al. 161

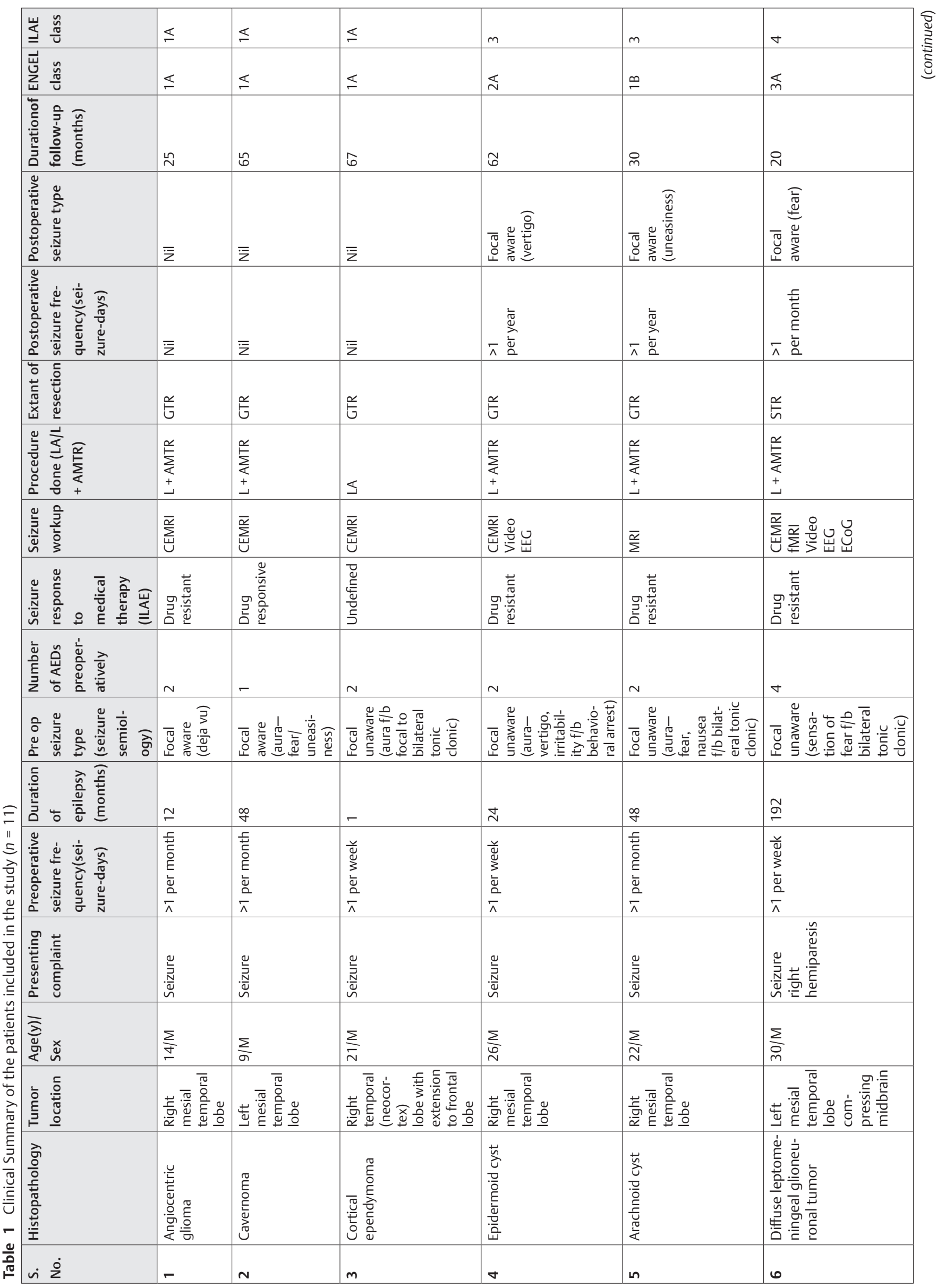




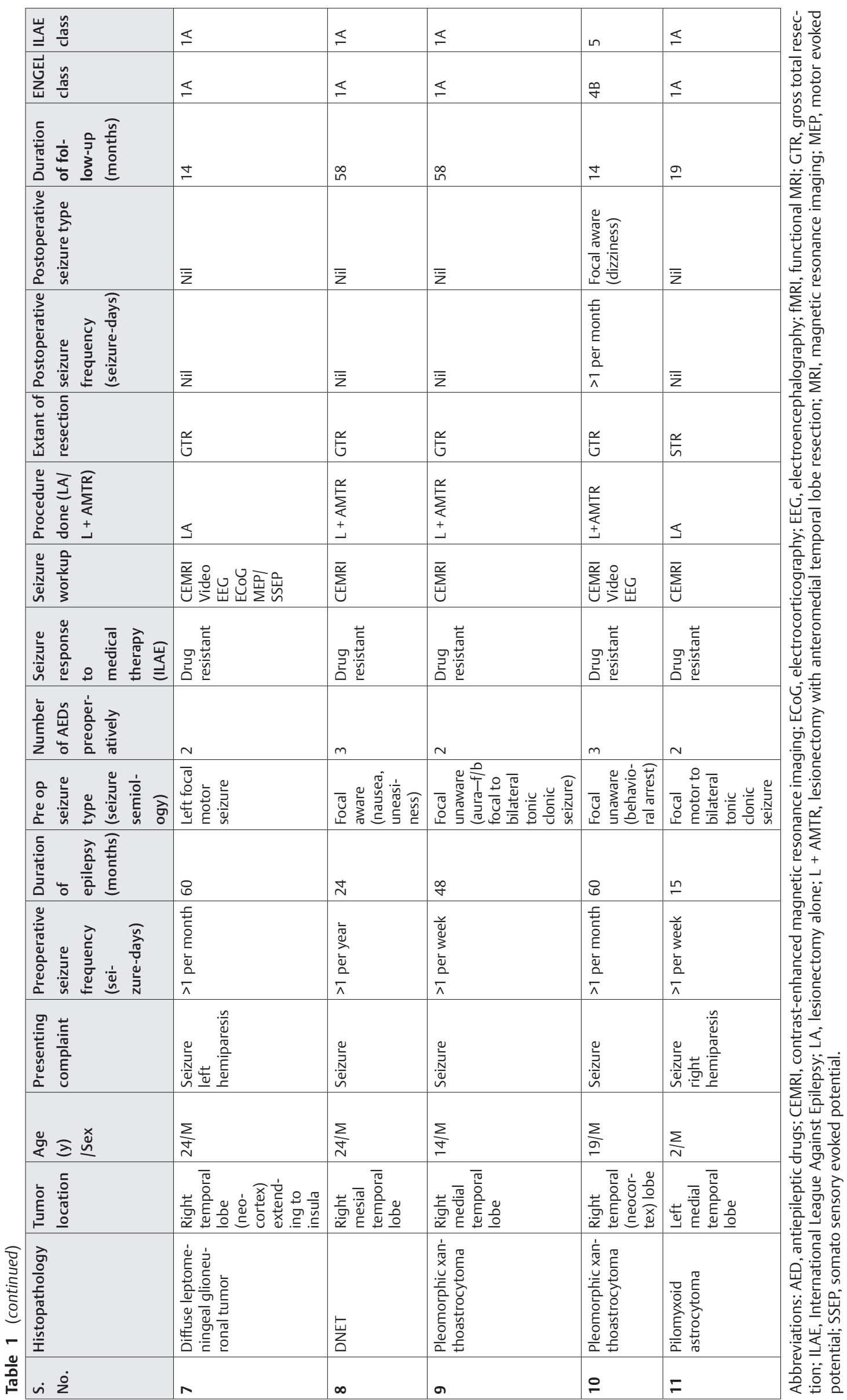


and the average duration of follow-up was 39.2 months. Three patients had a focal deficit (two cases of diffuse leptomeningeal glioneuronal tumor and one patient of pilomyxoid astrocytoma) preoperatively. All other patients had seizures as the only complaint. All except two patients had drug resistant epilepsy. Patients with cavernoma, cortical ependymoma, and pilomyxoid astrocytoma were operated primarily for the lesion. While seizure control was the primary indication for surgical intervention in all the other eight cases. As per the ILAE criteria for epilepsy surgery, in the presence of a lesion (like cavernoma, DNET, etc.), the cutoff for surgery can be reduced to less than 1 year. Three patients had focal aware seizures, two had focal motor onset seizures, and the rest all had focal unaware seizures. Seven patients were seizure free postoperatively, and the average age at diagnosis for these patients was 15.4 years (range 2-24 years). The average age of patients who had seizures postoperatively was 24.2 years (range 19-30 years). The duration of seizure in the postoperative seizure-free group was 29.7 months, and it was 81 months for the other group. Three patients had lesions on the left side. Two of these, cavernoma and DLGNT (case 6), had L + AMTR done. Preoperative functional MRI was done in the DLGNT case only. Standard epilepsy workup could not be done in the case of pilomyxoid astrocytoma as the patient was of only 2 years and needed urgent surgery for rapidly progressive hemiparesis and raised intracranial pressure features. It was not required in the case of cavernoma as the surgical trajectory did not require exposure of the speech zone. Three patients had temporal lobe lesions extending to nearby structures (insula, brainstem, and frontal opercula). Rest all patients had lesions confined to the temporal lobe or in associated with the temporal lobe only (epidermoid cyst, arachnoid cyst).

It was difficult to determine the histopathological diagnosis preoperatively for the gliomas and glioneuronal tumors based on MRI findings. Cavernoma, epidermoid cyst, arachnoid cyst, and DNET were the only pathologies that were correctly diagnosed preoperatively based on the clinicoradiological features. Preoperative video EEG was performed for cases with suspected MTS. It was done in the case of epidermoid and PXA (having a lesion in temporal neocortex while the seizure semiology was suggestive of mesial temporal lobe onset of seizure) as the seizure foci were ambiguous. It was confirmed to be in the temporal lobe on the same side of the lesion in both cases. Video EEG was also done in both the cases of DLGNT. As only single lesions were evident on MRI in all other cases, and there was no clinicoradiological discordance, EEG or ECoG was not done in them. Eight out of the 11 patients underwent lesionectomy with AMTR (L + AMTR), while 3 patients had LA done. Complete excision was achieved in nine cases. Patients with DLGNT (30 y/m) and pilomyxoid astrocytoma had subtotal excision. There were no new neurological deficits in any of the cases postoperatively except for a patient of DLGNT $(24 \mathrm{y} / \mathrm{m})$ who had tumor extension to the insula. Ten out of the 11 patients had improvement in their seizures, as either decreased or no seizures postoperatively. Two patients had seizures in the immediate postoperative period. Only one patient, the case of pleomorphic xanthoastrocytoma, had postoperative seizure frequency same as in preoperative status, suggesting no improvement (Engel class $4 \mathrm{~b}$ and ILAE class 5). This patient was a 19-year-old man with a history of intractable seizures for the last 5 years. On the MRI brain, the lesion was a well-defined, $2 \mathrm{~cm} \times 2 \mathrm{~cm} \times 2 \mathrm{~cm}$, located in the right temporal lobe with minimal edema, hypointense on T1, hyperintense on T2, with contrast enhancement. Lesionectomy with amygdalohippocampectomy was done. The AED had to be increased postoperatively. There is no residual lesion or recurrence on the follow-up scan. The second case with the same pathology, a 14-year-old boy, had a similar clinical picture but a better outcome. The patient had undergone lesionectomy with amygdalohippocampectomy. In 4 years of follow-up, the patient is seizure free (1a class in both Engel and ILAE).

One patient with a temporal epidermoid cyst had a decrease in seizure frequency, though focal seizures persisted (Engel class 2a and ILAE class 3). Two patients with diffuse leptomeningeal glioneuronal tumors were included in our series. The first patient was operated on with a differential diagnosis of mesial temporal cavernoma based on the radiological features ( - Fig. 2 A). Though the seizure semiology and MRI findings were concordant, video EEG was done to confirm the involvement of medial temporal structures as the lesion was on the left side. Intraoperative ECoG and deep electrodes were employed intraoperatively as the scalp EEG is less sensitive in deep-seated tumors like this case. It showed abnormal signal spikes from the inferomedial temporal lobe. Inferior temporal gyrus resection followed by lesionectomy and amygdalohippocampectomy was done. Subtotal excision could be achieved as the lesion was reaching the brainstem with loss of planes. Postoperatively, the patient has the same power of $4 / 5$ (both upper and lower limb) as in preoperative status. Histopathology was suggestive of DLGNT ( - Fig. 2B). In the 10 months of follow-up, there was a reduction in seizure frequency and he belonged to class 3a of Engel and 4 of ILAE classification. The second patient had also presented with intractable seizures of 5 years duration and hemiparesis. On MRI, the lesion was involving the temporal lobe and extending to the insula and measured $10 \mathrm{~cm} \times 6 \mathrm{~cm} \times 5 \mathrm{~cm}$. Preoperatively, video EEG was done as the seizure semiology was suggestive of seizure onset from the frontal lobe though the major part of the tumor was in the temporal neocortex. It was suggestive of seizure onset from the temporal lobe ipsilateral to the lesion with secondary spread to the ipsilateral frontal lobe. We operated upon him with a preoperative diagnosis of low-grade insular glioma with hemorrhage, possibly having conversion to the higher grade. Frontotemporal craniotomy with transcortical gross total excision of the tumor was done. As scalp EEG is less sensitive for deep lesions (seizure foci) like in insula, intraoperative ECoG was done. It was suggestive of the epileptic zone being confined to the temporal neocortex; hence, LA was done. The patient had hemiparesis post surgery despite the use of motor evoked potential (MEP) monitoring. The patient is presently seizure free (1a class in both Engel and ILAE). Another patient with an arachnoid cyst had reduced frequency with no loss of awareness 


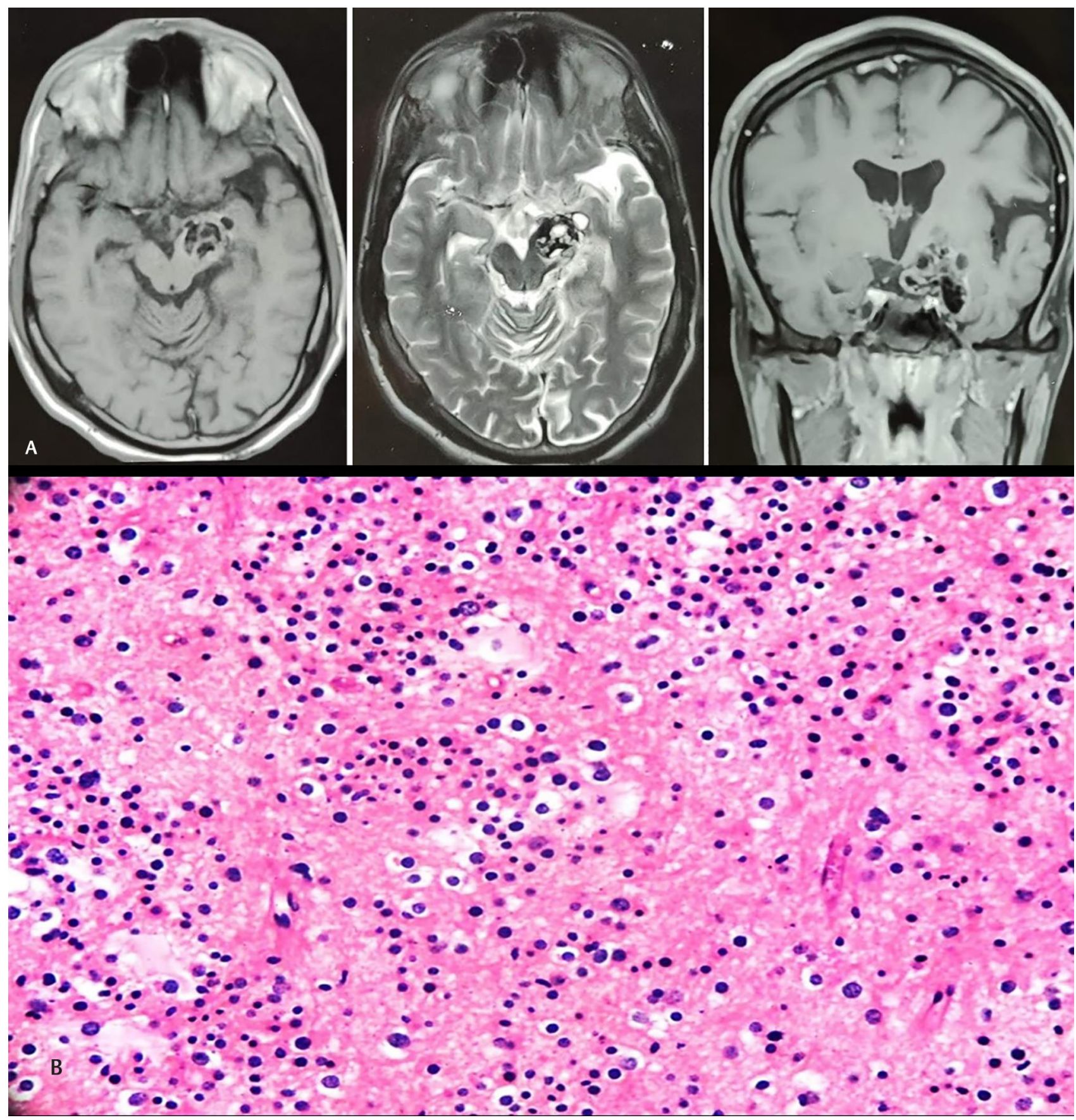

Fig. 2 (A) magnetic resonance imaging (MRI) head suggesting a well-defined solid cystic nodular lesion abutting medial temporal lobe with compression over midbrain (from left to right-T1 axial, T2 axial, and T1 + contrast-coronal images). (B) H\&E (Hematoxylin and eosin)-stained section showing a small focus of leptomeningeal tumor composed of sheets of round to polygonal cells with mildly isomorphic nuclei, clear cytoplasm, and interspersed thin capillaries at places. Mitosis/necrosis/endothelial proliferation were not seen. The adjacent parenchyma showed heavy calcification and few dystrophic neurons. Clusters of irregular thick-walled vessels were also seen in the meninges.

in the postoperative period. Rest all patients (7/11) were seizure free postoperatively.

Another patient with pilomyxoid astrocytoma was a 2-year-old male child with 15 months of intractable seizures and progressive right hemiparesis. The MRI showed a well-defined, intra-axial solitary lesion involving the medial temporal lobe. It was contrast-enhancing, T1-hypointense, and T2-hyperintense lesion. The patient underwent gross total excision of the tumor. Postoperatively, the patient is seizure free (1a class in both Engel and ILAE). The patient with cortical ependymoma was operated on with a differential diagnosis of a low-grade glioma on MRI. A gliotic rim was seen around the tumor which was completely excised. The histopathology confirmed intraparenchymal ependymoma ( - Fig. 3A-C). 


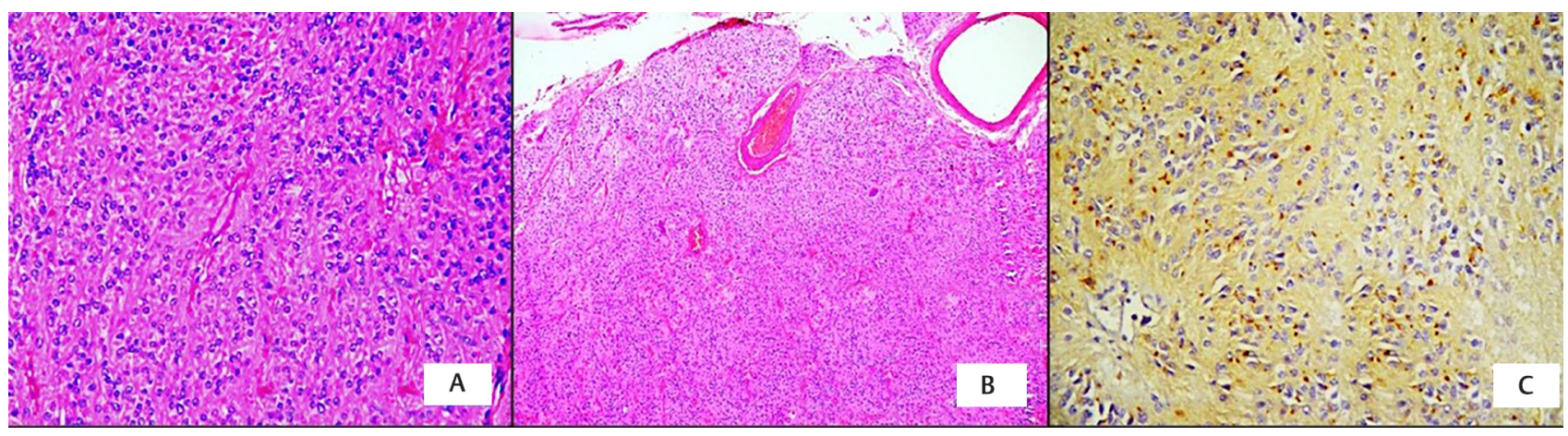

Fig. 3 (A and B) Cortical ependymoma with diffuse Infiltration of cortex by small monotonous glial cells with nuclear atypia embedded in the fibrillary background. A moderately cellular tumor, displaying tumor cells with round to oval nuclei, fine chromatin, and ill-defined cytoplasm forming perivascular pseudorosettes. Foci of the tumor had cells with pale clear cytoplasm. At places, pseudopapillary patterns were also noted. There was no evidence of necrosis, microvascular proliferation, or increased mitotic activity. Foci of calcification were also seen. (C) Dot-like immunoreactivity for epithelial membrane antigen (EMA) in cortical ependymoma.

\section{Discussion}

Mesial temporal sclerosis (MTS) is the most common etiology presenting as TLE. ${ }^{5,8}$ The other causes include traumatic brain injury, infections like meningitis, encephalitis, hypoxic-ischemic encephalopathy, stroke, genetic syndromes, and neoplastic lesions. Wolf et $\mathrm{al}^{6}$ had reported a series of 216 cases, with 75 patients having temporal lobe neoplasm causing seizures. Ganglioglioma (34) was the most common pathology followed by pilocytic astrocytoma(17), oligodendroglioma (9), diffuse astrocytoma (6), DNET (6), pleomorphic xanthoastrocytoma (1), and oligoastrocytoma (1). ${ }^{9}$ The nonneoplastic lesions included cavernoma (11), arachnoid cyst (1), and few other lesions. A study by Falah et al in the pediatric population found glioma (46\%) as the most common pathological lesion causing a seizure. ${ }^{10}$ Another study of 100 cases of temporal lobe epilepsy, reported that only $6 \%$ of patients had neoplastic lesions. ${ }^{5}$ All these studies implicated ganglioglioma or gliomas as the most common neoplastic lesions causing temporal lobe epilepsy. There were no cases of ganglioglioma in our series and the most common pathology was diffuse astrocytoma (4) after MTS (5). The seizure outcome in these studies was mainly dependent on these pathologies. The lesions which were reported as least common in these studies formed a significant portion of cases in our study as a cohort. This subgroup was heterogenous but had a similar clinical presentation and outcome on subgroup analysis. Though there are multiple case reports/series of these individual pathologies, they have not been evaluated as a single cohort previously.

The patients in this cohort were young and all males. This is similar to other studies that reported greater incidence in young patients and males. ${ }^{5-7}$ However, the average duration of epilepsy ( $\sim 4$ years) in these patients was less than other studies (7-12 years) ${ }^{6,7}$ The duration of symptoms is affected by the Engel/ILAE grades, with higher grades presenting earlier. Given the benign nature of tumors in this cohort, such a long duration of seizures is expected. Also, most of these tumors did not cause significant deficits that would make them present early. Most of these patients presented with complex partial seizures (8/11). Fifty percent (4) of patients with complex partial seizure had complete remission, while $37.5 \%$ (3) patients had reduced frequency and in one patient there was an increased AED requirement to maintain the same preoperative grade of seizures. All the patients with SPS were seizure free postoperatively. Seven (63.6\%) patients were seizure free postoperatively at an average of 29.27 months of follow-up. It is similar to the reports of Alsemari et al and Fallah et al (66.5\% at 5-year and 75\% at 2-year follow-up, respectively). ${ }^{10,11}$ These investigators had used intraoperative ECoG. Patients who had complete seizure freedom had a younger age at surgery and a shorter duration of symptoms. These same factors-younger age, shorter duration of symptoms, and focal seizures-had been shown to have a better prognosis by other authors. ${ }^{12-14}$ The analysis suggests that this heterogenous small subgroup of pathology at study has a clinical presentation, seizure outcome, and prognostic factors similar to cohorts of common pathologies causing temporal lobe epilepsy.

MRI brain was substantial in determining the extent of the lesion and possible involvement of the amygdala and hippocampus. However, it was difficult to determine the histopathology of most of the lesions on radiological features alone. Only 4 (epidermoid cyst, arachnoid cyst, cavernoma, and DNET) out of the 11 cases were correctly diagnosed on preoperative MRI. Lesionectomy was done when the only neocortex was involved. AMTR was done with lesionectomy if the lesion extended to mesial temporal structures. Cavernoma could be diagnosed well on MRI and it aids in deciding the extent of excision. In our case, it was a $3.3 \mathrm{~cm} \times 2.6 \mathrm{~cm}$ lesion that was involving the left temporal lobe. As it was extending to the medial temporal lobe with concordant semiology, and resection of the hemosiderin rim is recommended to prevent seizure persistence, lesionectomy with AMTR was done. Some studies, however, recommend excision of the cavernoma only, in case the dominant lobe is involved. ${ }^{15,16}$ The side of the lesion is a critical factor in decision-making to include AMTR with lesionectomy. The left TLE patients tend to perform worse than the right TLE patients on verbal memory, both before and after surgery. In most cases, the verbal memory is localized in the left hemisphere. Nonverbal memory deficits have been mostly associated with right temporal 
lobe resections. The occurrence of visual field defects, however, is not affected by the side of resection. Two out of the three patients with left-sided lesions underwent $\mathrm{L}+$ AMTR in this study. The postoperative memory and visual status of the patients were not different from preoperative findings on routine neurological assessment. However, the subtle changes could be evident on detailed neuropsychological and visual assessments.

The possible site of epileptogenic focus can be decided based on the MRI features. Three subtypes of DNET has been defined on MRI. These have been correlated with the possible epileptogenic foci, in and around them ( - Table 2).$^{17}$ This may aid in defining the extent of resection for DNET. Our patient had a "type 1" radiological subtype with a characteristic "bubbly pattern." A gross total resection with amygdalohippocampectomy was done. Postoperatively, there was no episode of seizure and all his AEDs has been gradually tapered in the last 4 years (1A class in Engel and $1 \mathrm{~A}$ in ILAE). In all the three patients with MRI showing extratemporal extension, seizure semiology, video EEG, and ECoG played a key role in deciding the epileptic focus and need for AMTR. In all these cases, we found the focus to be in the temporal lobe. Two attained seizure freedom on LA, while one patient undergoing L + AMTR had a good seizure outcome but persistent auras.

Persistent postoperative seizures were seen in patients with pleomorphic xanthoasctrocytoma (PXA), leptomeningeal glioneuronal tumor, epidermoid cyst, and arachnoid cyst. The possible cause of poor outcome in these patients could be the tumor-induced changes in the brain parenchyma. The cysts of the epidermoid cause local inflammatory response, leading to abnormal excitability and sclerosis. ${ }^{18}$ The arachnoid cyst has been associated with hippocampal agenesis. ${ }^{19,20}$ Similarly, low-grade tumors of the temporal lobe tend to induce palatogenesis in surrounding tissue including the hippocampus and amygdala by various mechanisms like inducing changes in neurotransmitters and their receptors, ionic changes, hypoxia, acidosis, immunologic changes, inflammatory changes, or morphological changes. ${ }^{21}$ The epidermoid cyst was related to the anterior and medial temporal lobe. So lesionectomy with AMTR was done. In the case of arachnoid cysts, one author concluded that the presence of an arachnoid cyst in the middle cranial fossa in their series of patients with seizures was incidental and they do not necessarily reflect the location of the seizure focus. ${ }^{22}$ However,

Table 2 Histological types of DNET with their MRI and epileptogenic zone corelation

\begin{tabular}{|l|l|l|}
\hline $\begin{array}{l}\text { Histopathological } \\
\text { type }\end{array}$ & $\begin{array}{l}\text { Corresponding } \\
\text { type on MRI }\end{array}$ & $\begin{array}{l}\text { Epileptogenic } \\
\text { foci location }\end{array}$ \\
\hline I-Simple & Type 1 (cystic) & Within the tumor \\
\hline II-Complex & Type 1 (cystic) & Peritumoral \\
\hline III-Nonspecific & $\begin{array}{l}\text { Type } 2 \text { (nodular) or } \\
\text { 3 (dysplastic) }\end{array}$ & $\begin{array}{l}\text { Extensively around } \\
\text { the tumor }\end{array}$ \\
\hline
\end{tabular}

Abbreviations: DNET, dysplastic neuroectodermal tumor; MRI, magnetic resonance imaging. most authors have found the middle cranial fossa arachnoid cyst to be linked to temporal lobe hypoplasia by an uncertain "progenitor-progeny" relationship. ${ }^{19,20}$ Our patient had a Galassi type III arachnoid cyst. Lesionectomy with AMTR was done. There was a decrease in seizure frequency but no complete remission. The patient continues to be on AED for seizure control. In these cases, we suspect either there was a residual epileptic zone/part of the network or the presence of a "dual pathology" that was not apparent on MRI. An intraoperative ECoG or a preoperative phase two seizure workup may have helped in these cases is finding the possible occult foci. The patient of DLGNT was older and had a long duration of seizures, and only subtotal excision was achieved. Patients with PXA also had a long duration of seizures while other poor prognostic factors were absent. Harrod et al had concluded five important reasons for a surgical failure (insufficient resection, relapse on the contralateral side, lateral temporal neocortical epilepsy, dual pathology, and extratemporal lobe epilepsy mimicking temporal lobe epilepsy or temporal plus epilepsy). ${ }^{23} \mathrm{~A}$ second-stage surgery involving amygdalohippocampectomy is safe and with better outcome. $^{24,25}$ Amygdalohippocampectomy was done in all these cases along with complete lesionectomy. Resection of the residual lesion in DLGNT would cause postoperative deficits. The patient had now only minor focal seizures (infrequent aura only). So, further surgical intervention was not done. Similarly, in other patients with some persistent postoperative seizures, only a minor aura was present. Further investigations for persistent seizure foci was offered to the patients, but it was denied as the patients had significant relief and the infrequent seizure did not hamper their daily activities.

\section{Limitation}

This is a retrospective study. The inherently small sample size precludes the application of statistical analysis and tests of significance. The subgroup is heterogeneous. However, the analysis of the clinical and outcome associated variables yielded similar results. The surgical intervention in different pathologies was guided by their preoperative MRI impression as per the operative surgeon. A detailed neuropsychological assessment and visual assessment would have allowed better evaluation of the possible adverse outcomes of LA versus L + AMTR.

\section{Conclusion}

Despite the small and heterogeneous nature of the subgroup of these uncommon lesions causing temporal lobe epilepsy, the clinical presentation, prognostic factors, and seizure outcome are similar to the cohort of common pathologies, including MTS. Tumors with significant peritumoral effects, long duration of seizures, and focal unaware seizures are poor prognostic factors. MRI may not differentiate between the pathology within the glioma subgroup, but the postoperative seizure outcomes remain the same irrespective of the histopathology. 


\section{Note}

The study was presented at Sanjay Gandhi Institute of Medical Sciences, Lucknow, in May 2019.

\section{Funding}

None.

\section{Conflict of Interest}

None declared.

\section{References}

1 Al Sufiani F, Ang LC. Neuropathology of temporal lobe epilepsy. Epilepsy Res Treat 2012;2012:624519

2 Kemerdere $\mathrm{R}$, Ahmedov ML, Alizada O, Yeni SN, Oz B, Tanriverdi T. Effect of temporal neocortical pathology on seizure freeness in adult patients with temporal lobe epilepsy. World Neurosurg 2018;116:e801-e805

3 Thom M, Bertram EH, Chapter 14-Temporal lobe epilepsy. In: Stefan H, Theodore WH, eds. Handbook of Clinical Neurology. Vol 107. Elsevier; 2012 225-240

4 Kwan P, Arzimanoglou A, Berg AT, et al. Definition of drug resistant epilepsy: consensus proposal by the ad hoc Task Force of the ILAE Commission on Therapeutic Strategies. Epilepsia 2010;51(6):1069-1077

5 Radhakrishnan VV, Rao MB, Radhakrishnan K, et al. Pathology of temporal lobe epilepsy: an analysis of 100 consecutive surgical specimens from patients with medically refractory epilepsy. Neurol India 1999;47(3):196-201

6 Wolf HK, Campos MG, Zentner J, et al. Surgical pathology of temporal lobe epilepsy. Experience with 216 cases. J Neuropathol Exp Neurol 1993;52(5):499-506

7 Jorge CL, Nagahashi-Marie SK, Pedreira CC, et al. Clinical characteristics and surgical outcome of patients with temporal lobe tumors and epilepsy. Arq Neuropsiquiatr 2000;58(4):1002-1008

8 Martinoni M, Berti PP, Marucci G, et al. Pathology-based approach to seizure outcome after surgery for pharmacoresistant medial temporal lobe epilepsy. World Neurosurg 2016;90:448-453

9 Casazza M, Avanzini G, Ciceri E, Spreafico R, Broggi G, Lesionectomy in epileptogenic temporal lobe lesions: preoperative seizure course and postoperative outcome. In: Ostertag CB, Thomas DGT, Bosch A, Linderoth B, Broggi G, eds. Advances in Stereotactic and Functional Neurosurgery Vol 12. Vienna, Austria: Springer; 1997 64-69
10 Fallah A, Weil AG, Sur S, et al. Epilepsy surgery related to pediatric brain tumors: Miami Children's Hospital experience. J Neurosurg Pediatr 2015;16(6):675-680

11 Alsemari A, Al-Otaibi F, Baz S, et al. Epilepsy surgery series: a study of 502 consecutive patients from a developing country. Epilepsy Res Treat 2014;2014:286801-286801

12 Ko A, Lee JS. Factors associated with seizure and cognitive outcomes after epilepsy surgery for low-grade epilepsy-associated neuroepithelial tumors in children. Clin Exp Pediatr 2020;63(5):171-177

13 Mohan M, Keller S, Nicolson A, et al. The long-term outcomes of epilepsy surgeryPLoS One $2018 ; 13$ (5):e0196274</jrn>

14 Mehrotra A, Singh S, Kanjilal S, et al. Factors affecting seizure outcome in Long-term epilepsy associated tumors (LEATs) in children and young adolescents. Clin Neurol Neurosurg 2020;197:106104

15 Sommer B, Kasper BS, Coras R, et al. Surgical management of epilepsy due to cerebral cavernomas using neuronavigation and intraoperative MR imaging. Neurol Res 2013;35(10):1076-1083

16 Moran NF, Fish DR, Kitchen N, Shorvon S, Kendall BE, Stevens JM. Supratentorial cavernous haemangiomas and epilepsy: a review of the literature and case series. J Neurol Neurosurg Psychiatry 1999;66(5):561-568

17 Chassoux F, Daumas-Duport C. Dysembryoplastic neuroepithelial tumors: where are we now? Epilepsia 2013;54(Suppl 9) :129-134

18 Hiraishi T, Oishi M, Kitaura H, et al. Epidermoid cyst involving the medial temporal lobe: surgical pathologic features of the epileptogenic lesion. Neuropathology 2012;32(2):196-201

19 Robinson RG. The temporal lobe agenesis syndrome. Brain 1964;87(1):87-106

20 Kobayashi E, Bonilha L, Li LM, Cendes F. Temporal lobe hypogenesis associated with arachnoid cyst in patients with epilepsy. Arq Neuropsiquiatr 2003;61(2B) :327-329

21 You G, Sha Z, Jiang T. The pathogenesis of tumor-related epilepsy and its implications for clinical treatment. Seizure 2012;21(3):153-159

22 Arroyo S, Santamaria J. What is the relationship between arachnoid cysts and seizure foci? Epilepsia 1997;38(10):1098-1102

23 Harroud A, Bouthillier A, Weil AG, Nguyen DK.. Temporal lobe epilepsy surgery failures: a review. Epilepsy Res Treat 2012;2012-201651

24 Jung R, Aull-Watschinger S, Moser D, et al. Is reoperation an option for patients with temporal lobe epilepsy after failure of surgery? Seizure 2013;22(7):502-506

25 Surges R, Elger CE. Reoperation after failed resective epilepsy surgery. Seizure 2013;22(7):493-501 